\title{
The Physics of Edge Resonant Magnetic Perturbations in Hot Tokamak Plasmas
}

T. E. Evans, K. H. Burrell, M. E. Fenstermacher, R. A. Moyer, T. H. Osborne, M. J. Schaffer, W. P. West, L. W. Yan, J. A. Boedo, E. J. Doyle, G. L. Jackson, C. J. Lasnier, A. W. Leonard, T. L. Rhodes, P. R. Thomas, J. G. Watkins, L. Zeng

June 16, 2006

Physics of Plasmas 
This document was prepared as an account of work sponsored by an agency of the United States Government. Neither the United States Government nor the University of California nor any of their employees, makes any warranty, express or implied, or assumes any legal liability or responsibility for the accuracy, completeness, or usefulness of any information, apparatus, product, or process disclosed, or represents that its use would not infringe privately owned rights. Reference herein to any specific commercial product, process, or service by trade name, trademark, manufacturer, or otherwise, does not necessarily constitute or imply its endorsement, recommendation, or favoring by the United States Government or the University of California. The views and opinions of authors expressed herein do not necessarily state or reflect those of the United States Government or the University of California, and shall not be used for advertising or product endorsement purposes. 


\section{THE PHYSICS OF EDGE RESONANT MAGNETIC PERTURBATIONS IN HOT TOKAMAK PLASMAS}

by

T.E. EVANS, K.H. BURRELL, M.E. FENSTERMACHER ${ }^{*}$, R.A. MOYER ${ }^{\dagger}$, T.H. OSBORNE, M.J. SCHAFFER, W.P. WEST, L.W. YAN ${ }^{\ddagger}$, J.A. BOEDO ${ }^{\dagger}$, E.J. DOYLE\#, G.L. JACKSON, C.J. LASNIER*, A.W. LEONARD, T.L. RHODES", P.R. THOMAS", J.G. WATKINS ${ }^{\S}$ and L. ZENG\#"

This is a preprint of a paper to be presented at the 47th Annual Meeting of Division of Plasma Physics, October 24-28, 2005, Denver, Colorado, and to be published in Phys. Plasmas.

*Lawrence Livermore National Laboratory, Livermore, California.

tUniversity of California, San Diego, California.

‡Southwestern Institute of Physics, Chengdu, Sichuan, China.

\#University of California, Los Angeles, California.

"CEA Cadarache EURATOM Association, Cadarache, France.

${ }^{\S}$ Sandia National Laboratory, Albuquerque, New Mexico.

Work supported by

the U.S. Department of Energy

under DE-FC02-04ER54698, W-7405-ENG-48(LLNL),

DE-FG02-04ER54758, DE-FG03-01ER54615, and DE-AC04-94AL85000

\section{GENERAL ATOMICS PROJECT 30200 OCTOBER 2005}





\section{The physics of edge resonant magnetic perturbations in hot tokamak plasmas}

T.E. Evans, ${ }^{\text {a) }}$ K.H. Burrell, ${ }^{\text {a) }}$ M. E. Fenstermacher, ${ }^{\text {b) }}$ R.A. Moyer ${ }^{\text {c) }}$ T.H. Osborne, ${ }^{\text {a) }}$ M.J. Schaffer, ${ }^{\text {a) }}$ W.P. West, ${ }^{\text {a) }}$ L.W. Yan, ${ }^{\text {d) }}$ J.A. Boedo, ${ }^{\text {c) }}$ E.J. Doyle, ${ }^{\text {e) }}$ G.L. Jackson, ${ }^{\text {a) }}$

C.J. Lasnier, ${ }^{\text {b) }}$ A.W. Leonard, ${ }^{\text {a) }}$ T.L. Rhodes, ${ }^{\text {e) }}$ P.R. Thomas, ${ }^{\text {f) J.G. Watkins }}{ }^{\text {g) }}$ and L. Zeng

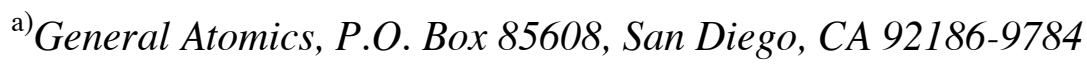

${ }^{b)}$ Lawrence Livermore National Laboratory, Livermore, California, USA

${ }^{c}$ University of California, San Diego, California, USA

${ }^{\mathrm{d})}$ Southwestern Institute of Physics, Chengdu, Sichuan, China

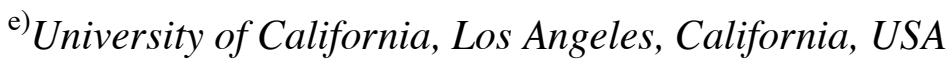

${ }^{\mathrm{f})}$ CEA Cadarache EURATOM Association, Cadarache, France

${ }^{g}$ Sandia National Laboratory, Albuquerque, New Mexico, USA

\section{ABSTRACT}

Small edge resonant magnetic perturbations are used to control the pedestal transport and stability in low electron collisionality $\left(v_{e}^{*}\right)$, ITER relevant, poloidally diverted plasmas. The applied perturbations reduce the height of the density pedestal and increase its width while increasing the height of the electron pedestal temperature and its gradient. The effect of the perturbations on the pedestal gradients is controlled by the current in the perturbation coil, the poloidal mode spectrum of the coil, the neutral beam heating power and the divertor deuterium fueling rate. Large pedestal instabilities, referred to as edge localized modes (ELMs), are completely eliminated with radial magnetic perturbations $\left(\delta b_{r}^{(m / n)}\right.$ ) at the $q=m / n=11 / 3$ surface exceeding $\delta b_{r}^{(11 / 3)} B_{\phi}^{-1}=2.6 \times 10^{-4}$ where $B_{\phi}$ is the toroidal magnetic field on axis. The resulting ELM-free H-mode plasmas have stationary densities and radiated power that have been maintained in DIII-D for up to $2550 \mathrm{~ms}$ 
(17 energy confinement times) and are limited only by hardware constraints. It is found that changes caused by the magnetic perturbations in the pedestal profiles cannot be explained by a straightforward application of stochastic quasi-linear diffusion theory due to the complex nature of the transport physics involved when boundary layer field lines connect regions of hot plasma directly to material surfaces.

PACS numbers: 28.52-s, 52.55.Fa, 52.55.Ra 


\section{INTRODUCTION}

Non-axisymmetric magnetic perturbations from a variety of sources are common in toroidal magnetic confinement devices. They originate in part from time varying plasma currents driven by magnetohydrodynamical (MHD) modes [1] and tearing instabilities on rational flux surfaces [2], thermoelectric currents flowing on open field lines [3], coherent modes [4], edge harmonic oscillations [5] and edge localized modes (ELMs) [6] as well as an array of higher frequency electromagnetic waves and turbulence [7]. Outside the plasma, sources such as field-errors from asymmetries in toroidal and poloidal magnetic field coils, magnetic materials, vacuum vessel image and return currents [8], external control coils used to stabilize plasma modes, and correction coils [9] used to minimize perturbations from known field-errors on low integer rational surfaces all contribute to the structure of the magnetic field in which the plasma resides. At the edge of the plasma where the safety factor $[q(\psi)$ defined as the rate of change in toroidal magnetic flux with poloidal magnetic flux $\psi$ ] increases rapidly, all of these perturbations contribute to the creation of closely spaced resonant magnetic islands which may result in the formation of edge stochastic layers. In a poloidally diverted tokamak, the high magnetic shear $\partial q / \partial \psi$ across the edge of the plasma results in an increased density of island states and a significantly higher probability of forming open stochastic layers that connect magnetic field lines to plasma facing material surfaces [10]. Thus, the edge plasma is immersed in a dynamically complex magnetic topology over the same region where substantial radial flows of mass and energy, driven by large gradients, compete with strong turbulent transport in highly sheared toroidal and poloidal plasma flow fields. Large radial gradients in the plasma pressure are often established by a strong reduction in turbulent 
transport due to sheared plasma flow. The large edge gradients are a key factor in the establishment of good confinement levels that make the tokamak the leading candidate for fusion reactors. However, they also lead to the instabilities known as ELMs which drive impulsive energy losses that can be detrimental to plasma facing surfaces. It is easy to understand why the development of tools to control the pedestal transport and stability is a compelling issue for improving the performance and operational safety of high energy density tokamak based fusion confinement systems.

A strong motivation for understanding the physics of edge stochastic layers is to enable the development of predictable and reliable tools for controlling key fusion plasma pedestal processes such as: the plasma temperature and pressure at the top of the pedestal, the size and frequency of ELMs, the energy and particle exhaust rate in steady-state conditions, the properties of boundary layer flows and momentum transport and the degree of edge impurity screening. A fundamental element in this line of research is acquiring a better understanding of how the plasma responds to an applied edge resonant magnetic perturbation (RMP). This requires the development of a practical model for the energy and particle transport through low collisionality $v_{e, n e o}^{*}=q_{95} R \varepsilon^{-3 / 2} \lambda_{e}^{-1}[11]$, stochastic, pedestal plasmas where $R$ is the major radius, $\varepsilon \equiv(a / R)$ is the inverse aspect ratio, $a$ is the minor radius and $\lambda_{e}\left(=\mathrm{v}_{T e} \tau_{e}\right.$, the product of electron thermal velocity $\mathrm{v}_{T e}$ and collision time $\tau_{e}$ ) is the mean free path for electron collisions. Here, $\lambda_{e}$ may be expressed in SI units as $\lambda_{e}=8.5 \times 10^{21}\left(T_{e}^{2} / n_{e}\right)(\mathrm{m})$ with $T_{e}$ given in units of $\mathrm{keV}$. Thus, we see that the resulting neoclassical collisionality $v_{e, \text { neo }}^{*}$ is proportional to $n_{e} T_{e}^{-2}$. 
Initial $n=3$, odd parity, I-coil perturbation experiments were found to be very effective for suppressing large Type-I ELMs in high collisionality $v_{e, \text { пео }}^{*}=1.0 \rightarrow 1.1$ plasmas with lower triangularities of $\sim 0.6-0.7$ [12]. In this configuration, the I-coil produced weak pedestal resonances $(\sim 1-2 \mathrm{G})$ with approximately the same amplitude as the DIII-D field-errors. Based on vacuum field line modeling, these resonances resulted in small, well isolated, magnetic islands with a narrow stochastic layer located at the foot of the pedestal [13]. Experimentally they had little or no effect on the pedestal profiles, $\mathrm{H}$-mode transport barrier, the depth or location for the edge $\mathrm{E}_{\mathrm{r}}$ well or on the overall quality of the plasma confinement although the toroidal rotation across the entire plasma was significantly reduced after a $200-300 \mathrm{~ms}$ decay time $[13,14]$. It is interesting to note that in these high $v_{e, \text { neo }}^{*}$ experiments the effects of the I-coil perturbation were maximized at the same resonance condition, $q_{95}=11 / 3$, as in low $v_{e, \text { neo }}^{*}$ lower single null plasmas and that the odd parity I-coil configuration produces a minimum in the spectrum amplitude across the pedestal [13] as opposed to the low $v_{e, \text { neo }}^{*}$ case with even parity which produces a maximum in the pedestal spectrum when $q_{95}=11 / 3$. In the high $v_{e, \text { neo }}^{*}$ ELM control experiments, ELMs appear to be stabilized by increasing small, high frequency, edge fluctuations which are believed to inhibit the onset of the large Type-I ELMs $[13,14]$.

In low $v_{e, \text { neo }}^{*}$, even parity, $n=3 \mathrm{RMP}$, experiments [15] the pedestal profiles are significantly altered by the I-coil perturbation and ELMs are stabilized when the total pedestal pressure gradient falls below the stability boundary for intermediate $n$ peelingballooning (P-B) modes [16-18]. Results from these low $v_{e, \text { neo }}^{*}$ ELM control experiments demonstrate that as the total pedestal pressure gradient falls below the peeling-ballooning P-B stability boundary ELMs are completely eliminated and the divertor $D_{\alpha}$ recycling signals become very quiet just as in QH-modes [15,19]. While changes observed in the pedestal pressure profiles during these low $v_{e, \text { neo }}^{*}$ RMP experiments are consistent with an 
increase in transport due to the formation of an open field stochastic magnetic boundary layer, it is surprising to find that the pressure profile changes are due to a larger than expected change in the global particle balance with only a modest $\sim 10 \%$ increase in the energy transport [20]. Expectations based on quasi-linear theory [21] suggest that the thermal transport should be enhanced significantly more than the particle transport. The observed changes in the global particle balance might be due to a change in the particle convection (e.g., the inward pinch) which is not included in the quasi-linear formulation.

In the following we focus on how externally applied edge RMPs change the DIII-D pedestal plasma and the effect these changes have on the edge MHD stability in strongly pumped, low $v_{e, \text { neo }}^{*}$, lower single null DIII-D H-mode plasmas. In Section II we provide an overview of the experimental set up. Section III summarizes the experimental results while Section IV provides a representative sampling of field line modeling results for several discharges used during the experiments described in Section III. A discussion of the interpretation of the results given in Sections III and IV is provided in Section V which is followed by a summary and conclusion section. 


\section{EXPERIMENTAL OVERVIEW}

In this paper we describe results from edge resonant magnetic perturbation (RMP) experiments in low $v_{e, \text {,neo }}^{*}=0.12 \rightarrow 0.04$ plasmas where both the DIII-D I-coil [22], configured for $n=3$ toroidal mode number perturbations, and the DIII-D correction coil (C-coil) operated in $n=1$ field-error feedback mode [23] were used simultaneously. The plasmas used in these experiments are configured for strong lower single null divertor pumping, as shown in Fig. 1(a), with a plasma current $I_{p} \approx 1.5 M A$ and a toroidal magnetic

field on axis $B_{\phi} \approx 2.0 T$. The injected neutral beam power $\left(P_{N B I}\right)$ was controlled to maintain a constant normalized plasma beta $\left(\beta_{N}\right)$ using the DIII-D plasma control feedback system. During these experiments $\beta_{N}$ was varied from $\beta_{N}=1.4$ to $\beta_{N}=2.2$ in order to assess how the RMP affected the pedestal profiles and ELM suppression as a function of plasma pressure and collisionality. In general, these plasmas where typical H-mode discharges with ITER 98(y2) scaling factors of approximately 1.0, elongations of $\sim 1.8$ and lower triangularities of $\sim 0.36$.

A 3D representation of the I-coil geometry, originally designed for MHD mode control in DIII-D [22], is shown in figure 1(b). The I-coil segments, shown above and below the mid-plane in Fig. 1(a), are located inside the DIII-D vacuum vessel just behind the graphite tiles that form the primary plasma facing material surface and are composed of 12 single turn loops. For the sake of simplicity, the DIII-D vacuum vessel is not shown in this figure. When viewing the coil inward toward the plasma, a positive I-coil current is defined to be in the counter-clockwise direction producing a perturbation field $\left(\delta b_{r}^{(m / n)}\right)$ that points in the direction of positive major radius $R$. The up/down parity of the I-coil is referred to as "even" when the upper and lower coil segments produce a $\delta b_{r}^{(m / n)}$ that is in 
the same direction at each toroidal angle $\phi$ and "odd" when the directions of $\delta b_{r}^{(m / n)}$ are opposite. When operated in the $n=3$ configuration, as shown in Fig. 1(b), the effect of the perturbing field is maximized across the pedestal region and strongly diminished in the core plasma. Since the $n=3$ configuration is three-fold symmetric, the perturbation produced by the I-coil can be rotated toroidally by $\phi=\pi / 3$ with respect to the other perturbing fields, e.g., field-errors from various sources and the field from the C-coil, composed of six window-frame loops centered on the mid-plane outside the vacuum vessel as shown in Fig. 1(a), and arranged in a dominant $n=1$ configuration.

In the experiments discussed here the I-coil is operated in the $n=3$ even parity configuration as shown in Fig. 1(b) while the C-coil is operated in the $n=1$ field-error correction mode. With this configuration, $n=1$ and $n=3 \mathrm{C}$ - and I-coil spectral components combine with $n=1, n=2$ and $n=3$ field-errors components to produce a relatively dense set of vacuum magnetic islands embedded in a strongly stochastic boundary layer. An example of the spectral features produced by these sources for the discharge discussed in this paper is given in Fig. 2. Here, Fig. 2(a) shows the $n=1$ part of the poloidal mode spectrum produced by the C-coil (with a current of $12.4 \mathrm{kA}$-turns) combined with the measured [8] $n=1$ field-errors at $\mathrm{t}=3.0 \mathrm{~s}$ in DIII-D discharge 123301. White contour lines are drawn for each $10 \%$ change in amplitude and the maximum mode amplitude $(15.1 \mathrm{G})$ is indicated in red while the minimum is in black. The color bar on the right indicates the amplitude scale in units of Gauss. The black dashed curve, given by $m=n q$ where $q$ is the safety factor, represents the location in normalized poloidal flux $\left(\psi_{N}\right)$ of the mode resonance contour while the solid black dots located along this curve represent the locations of the discrete spectral resonance such as $m / n=4 / 1$ at $q=4$ located on the $\psi_{N} \approx 0.97$ surface. The other resonances shown in Fig. 2(a) represent the $m / n=3 / 1$, and $m / n=2 / 1$ spectral components located at $\psi_{N} \approx 0.87$ and $\psi_{N} \approx 0.62$ respectively. Here, we see that the $n=1$ resonant modes are well 
separated in $\psi_{N}$ and have approximately equal amplitudes lying between the green and blue contours, i.e., $\sim 6-7 \mathrm{G}$.

Figure 2(b) shows the $n=3$ part of the poloidal mode spectrum which is produced with a current of $3.2 \mathrm{kA}$ in the I-coil and the measured DIII-D field-errors. Here, we see that all of the resonant modes $(m / n=10 / 3 \rightarrow 14 / 3)$ located across the pedestal region $0.92 \leq \psi_{N} \leq 1.0$ have amplitudes of approximately $6 \mathrm{G}$ and are quite closely spaced. We also note that the amplitude of the resonances located deeper inside the plasma $(m / n=9 / 3 \rightarrow 6 / 3)$ fall off rapidly with decreasing $\psi_{N}$ from $\sim 5.5 \mathrm{G}$ on the $q=3$ surface to $3.5 \mathrm{G}$ on the $q=2$ surface. It should be pointed out that the DIII-D field-errors also produce small $n=2$ resonances which are not shown in Fig. 2 but as discussed below do contribute to the level of stochasticity across the pedestal. The data shown in Fig. 2 corresponds to a safety factor at the $95 \%$ normalized flux surface $\left(q_{95}\right)$ of $11 / 3$ and as discussed below, this $q_{95}$ value corresponds a point in the experimental parameter space where the I-coil perturbation results in a complete elimination of the ELMs. In addition, as seen from Fig. 2(b) this operating point is located on the lower edge of the ridge in the $n=3$ pedestal resonance structure (the high red region located just above the resonance curve in the pedestal) which results in the largest magnetic island overlap and strongest level of edge stochasticity.

Figure 3 shows the relative widths and positions the magnetic islands produced by the resonant spectral components shown in Fig. 2. Islands produced by $n=2$ resonances (not shown if Fig. 2) are included in this figure since they can sometimes close gaps between islands produced by other sources and broaden the stochastic layer. We see from this figure that the islands from the $q=3$ surface all the way to separatrix at $\psi_{N}$ are strongly overlapping (with the $m / n=7 / 2$ island filling a gap between the $m / n=10 / 3$ and $m / n=11 / 3$ islands). The island width calculations shown in Fig. 3 are based on the vacuum field spectral amplitudes given in Fig. 2 and do not include a plasma response 
such as image currents driven in the plasma by flows [24,25] or bootstrap currents produced by changes in the plasma pressure distributions near magnetic islands [26]. These effects are expected to be important in low collisionality, rapidly rotating, plasmas such as those discuss in this paper and may cause significant changes in the island widths and the degree of island overlap i.e., the width and strength of the stochastic layer. Since effects such as these are difficult to quantify in the present experiments and theories dealing with these issues have not yet been fully developed for the pedestal region of the plasma, we defer the discussion of these issues until more data is available. 


\section{EXPERIMENTAL RESULTS}

Over the last 15 years an extensive set of resonant magnetic perturbation experiments have been carried out in DIII-D using a variety of non-axisymmetric coil sets including the original $n=1$ coil used to study the effect of field-errors [27], the field-error correction coil i.e., the C-coil [23] and the internal I-coil [22] designed to provide resistive wall mode stabilization in DIII-D. Of the various coils used, only the I-coil is capable of producing the type of mode spectrum needed to control the pedestal i.e., the spectrum shown in Fig. 2(b) with a high density of large amplitude modes spanning the pedestal region.

A typical low $v_{e, n e o}^{*}$, even parity, $n=3$ RMP discharge is shown in Fig. 4. In this discharge, an I-coil current of $3 \mathrm{kA}$, corresponding to a normalized $m / n=11 / 3$

perturbation $\delta b_{r}^{(11 / 3)} B_{\phi}^{-1}=2.6 \times 10^{-4}$ where $B_{\phi}=2.0 T$, is turned on at $1.5 \mathrm{~s}$, as shown in Fig. 4(a), shortly after the transition to an H-mode. As shown in Fig. 4(b), the ELMs become less frequent and disappear as $q_{95}$ drops below $\sim 3.7$. We also note that line integrated electron density $\left(\bar{n}_{e}\right)$ and the pedestal density ( $\left.n_{e, p e d}\right)$, shown in Fig. 4(c), remain constant until the ELMs are completely eliminated and then slowly drop to a new equilibrium at about $3.0 \mathrm{~s}$. The average neutral beam injection (NBI) heating power and the total radiated power are shown in Fig. 4(d). In these experiments the NBI power was regulated by the plasma control system to keep a constant beta normal $\left(\beta_{N}\right)$. In this discharge $\beta_{N}=2.2$ requiring an NBI heat power of 7.9 MW during the I-coil pulse. In these experiments the average NBI power increased by $\sim 10-15 \%$ during the first part of the I-coil pulse while ELMs were still present and $q_{95}$ was above the resonant window needed for complete island overlap across the pedestal. As seen in Fig. 4, once $q_{95}$ reaches the resonant value the NBI power and the total radiated power remain relatively constant. Discharges of this type have been run up to an average NBI power of $10 \mathrm{MW}$ with no sign of a saturation in this behavior. 
A closer look at the detailed evolution of the pedestal parameters in this 7.9 MW discharge is of interest for understanding how the transport is affected by the edge RMP. Figure 5(a) shows how the normalized electron pedestal temperature $\left(\hat{T}_{e, p e d}\right)$, electron density $\left(\hat{n}_{e, p e d}\right)$ and electron pressure $\left(\hat{p}_{e, p e d}\right)$ evolve during the off-resonance part of the $q_{95}$ evolution compared to the in-resonance phase. Here, the pedestal parameters shown in Fig. 5(a) have been individually normalized by a constant factor at $t=1.9 \mathrm{~s}$ where $q_{95}=3.7$. A divertor $D_{\alpha}$ recycling signal is shown in Fig. 5(b) for reference. We see that during the off-resonance part of the I-coil pulse $(1.5 s \leq t \leq 1.9 s)$, while ELMs are still present, the pedestal parameters remain relatively constant. Once the resonance condition is satisfied $\hat{n}_{e, p e d}$ drops and $\hat{T}_{e, p e d}$ increases. Initially $\hat{n}_{e, p e d}$ drops slightly faster than $\hat{T}_{e, p e d}$ increases causing a drop in the $\hat{p}_{e, p e d}$. At about the same time as the resonance condition is satisfied the ELMs begin to disappear implying that the radial transport driven by the ELMs has been replaced by another mechanism which preferentially enhances particle over energy transport. A similar behavior is seen at other NBI heating powers. Using the same plasma conditions as those in Fig. 5, we reduced the average NBI heating power to 4.7 MW. At this lower power the ELM frequency starts to drop as $q_{95}$ crosses 3.7 but ELMs persist until $2.5 \mathrm{~s}$ in this case. Since these are repeat discharges the degree of vacuum island overlap and the level of open field stochasticity are the same. The key difference here is that $T_{e, p e d}$ is lower because of the reduced NBI heating power and $\lambda_{e, p e d} \propto T_{e, p e d}^{2} / n_{e, p e d}$ is somewhat smaller which, in principle, reduces the effectiveness of the stochastic layer as discussed in Section I. The pedestal parameters evolve in approximately the same way as they did in the $7.9 \mathrm{MW}$ case but over a longer time scale. This behavior is consistent with a reduction in the effectiveness of the open field stochastic layer at the lower $T_{e, p e d}$. As $T_{e, p e d}$ continues to increase, it appears that the increasing effectiveness of the stochastic layer transport i.e., increasing $\lambda_{e, p e d}$, overcomes the ELM driven transport and the ELMs are eliminated. Shortly after the ELMs are suppressed in the 4.7 MW case, $\hat{T}_{e, p e d}$ stabilizes and $\hat{n}_{e, p e d}$ continues to drop somewhat. 
This may imply that the particle transport is responding to changes other than an increase in the effectiveness of the open field stochastic layer.

A comparison of the $n_{e}, T_{e}$ and $p_{e}$ edge profiles during the RMP assisted ELM-free state in these two cases is shown in Fig. 6. Here, we see that a higher value of both $n_{e, p e d}$, Fig 6(a), and $T_{e, p e d}$, Fig. 6(b), in the 7.9 MW case results in an increase in $p_{e, p e d}$ Fig. 6(c). This comparison shows that a $70 \%$ increase in the NBI power, about $10 \%$ of which goes into an increase in the total radiated power, produces approximately a $20 \%$ increase in both $n_{e, p e d}$ and $T_{e, p e d}$ at the shifted pedestal symmetry point. Since the total stored energy increases by $30 \%$, the implication is that $\chi_{e, p e d}$ is relatively unaffected or slightly reduced by the increase in heating power assuming all the excess heating power $(\sim 30 \%)$ is lost through the electron conduction channel. Additionally, we believe the radial outward shift seen in the 7.9 MW pedestal profiles is a not an artifact of mapping the profile to the normalized poloidal flux surfaces but a consequence of the increased NBI input power since it is also seen as an outward shift in the R,Z coordinate space of the Thomson scattering system. These shifts are often observed during the I-coil RMP experiments and in some cases can be of order 1-2 $\mathrm{cm}$ at the Thomson location. The cause of these shifts is presently under investigation.

In order to better quantify the effect of the poloidal mode spectrum on the edge stochastic layer several discharges were run with current in only the lower I-coil loops. As discussed below, this reduces the spectral amplitudes of the $n=3$ I-coil resonance across the pedestal by more than a factor of two and is expected to have an observable effect on the radial transport and pedestal profiles. In these discharges, $I_{p}$ and $q_{95}$ are held constant starting at $1.8 \mathrm{~s}$ when $q_{95}$ reaches the 3.7 resonant value and a $3 \mathrm{kA}$ I-coil current is turned on at $t=2.0 \mathrm{~s}$. The $\beta_{N}$ feedback system quickly responds to the I-coil pulse and an immediate change in the ELM behavior, by briefly ( 100 ms) commanding a $20 \%$ increase in the average NBI heating power. Both $\bar{n}_{e}$ and $n_{e, p e d}$ immediately 
begin to decay to a new equilibrium with an e-folding time of $\sim 120-140$ ms. During the RMP assisted ELM-free phase $\beta_{N}$ feedback holds the average NBI power relatively stable at 7.9 MW with $\beta_{N} \approx 2.0$.

Figure 7(a) shows a comparison of the $n_{e, p e d}$ evolutions for repeat discharges with: 1) no I-coil current, 2) both the upper and lower I-coil loops and 3) only the lower I-coil loops. The I-coil currents in the upper loops are shown in Fig. 7(b) for each of these cases, and the ELM response is shown in Fig. 7(c,d,e) respectively for each of these cases. In the full I-coil case, ELMs are completely eliminated after a brief $\sim 180 \mathrm{~ms}$ relaxation period during which time the pedestal profiles adjust to the RMP. In the case with only the lower I-coil loops, ELMs are still present in the pedestal but have a higher frequency and are more symmetric in time than their predecessors prior to the I-coil pulse. Their peak amplitude is also reduced by as much as a factor of 5 compared to the no I-coil case shown in Fig. 7(c) where some of the peaks have been clipped to show differences in the baseline evolution.

It is interesting to note that the $n_{e, p e d}$ evolution of the $1 / 2$ and full I-coil cases are somewhat similar while in the I-coil off case has a $n_{e, p e d}$ that is significantly higher, particularly in view of the fact that the neutral pressure in the cryopump is less affected during the 1/2 I-coil pulse than during the full I-coil pulse. A comparison of the $n_{e, p e d}$, $T_{e, p e d}$ and $p_{e, p e d}$ profiles averaged over $2.61 s \leq t \leq 3.01 s$ for these three cases is shown in Figs. 8(a), 8(b) and 8(c) respectively. Profiles for the two cases with ELMs were generated using only Thomson scattering data for time slices just before an ELM onset. Based on this data, we see that the $n_{e, p e d}$ is strongly reduced in both the $1 / 2$ and full I-coil cases while $T_{e, p e d}$ is essentially unaffected in the 1/2 I-coil case and either shifted radially outward or increased in the full I-coil case. This may imply that the instability responsible for ELMs in these particular plasmas is more sensitive to changes in the $T_{e, p e d}$ profile than in the $n_{e, p e d}$ profile. It is interesting to note that $p_{e, p e d}$ is higher 
between $\psi_{N}=0.96$ and $\psi_{N}=1.0$ in the full I-coil case than in the $1 / 2$ I-coil case and have similar electron pedestal pressure gradients, $\nabla p_{e, p e d}$ which are both well below $\nabla p_{e, p e d}$ in the no I-coil case yet one has ELMs while the other does not.

The final comparison to be discussed is one in which we introduced a continuous deuterium gas feed into the lower divertor private flux region in order to increase $n_{e, p e d}$. The discharge evolution for this case is shown in Fig. 9. In these discharges, the divertor gas valve is turned on at $t=1.8 \mathrm{~s}$ and the ELM frequency prior to the I-coil pulse is reduced. Three discharges with increasing puff rates are compared. Figure 10(a) shows how $n_{e, p e d}$ changes with an 8.0 Torr-liter/s puffing rate (lower trace - 122489), a 20.4 Torr-liter/s puffing rate (middle trace - 122490) and a 39.2 Torr-liter/s puffing rate (upper trace - 122492). These discharges all had the same I-coil current, as shown in Fig. 10(b), but at the highest puffing rate small grassy ELMs were destabilized as $n_{e, p e d}$ approached $3 \times 10^{19} \mathrm{~m}^{-3}$, Fig. 10(e). While the grassy ELM may contribute somewhat to this difference, it appears that the global particle balance is strongly affected by a mechanism that prevents neutrals from getting into the plasma in the high puffing rate case compared to the intermediate puffing rate case.

Figure 11 provides comparisons between $n_{e}, T_{e}, p_{e}$ and $\nabla p_{e}$ profiles averaged between $2.91 s \leq t \leq 3.1 s$ for each of the 3 puffing rates. A fourth profile, taken just before an ELM in the same time window, during discharge 123302 with no I-coil, is also included as a reference. We note that as the puffing rate increases $n_{e, p e d}$ increases, Fig. 11(a), and $T_{e, p e d}$ decreases, Fig. 11(b). Figure 11(c) show a monotonic increase in $p_{e, p e d}$ with puffing rate although the change in this profile from 20.4 Torr-liter/s to 39.2 Torr-liter/s is quite modest. We see from Fig. 11(d) that $\nabla p_{e, p e d}$ is similar for all the puffing cases and well below that in the ELMing discharge. It is notable that the $n_{e}$ profile at the foot of the pedestal broadens significantly with increasing puffing rate indicating an increase in particle transport with puffing rate. 



\section{FIELD LINE MODELING}

In this section we compare field line integration modeling results from the TRIP3D code [10] for two of the discharges discussed above, one of which had current in both the upper and lower I-coil loops (123301) and the other having current in only the lower I-coil loops (123300). An example of the Pioncaré representation showing the field line distribution on a poloidal plane is given in Fig. 12. Figure 12(a) shows an R,Z space representation of the field line distribution along with the DIII-D vessel wall (solid line) and internal poloidal flux surfaces (dashed lines). The thick solid line that forms the outer surface of the field line puncture points (the densely packed solid region extending inward to just beyond the first dashed surface) is an invariant manifold that forms $1 / 2$ of a structure referred to as a homoclinic tangle [28]. In the presence of non-axisymmetric perturbation, the separatrix of a poloidally diverted plasma splits into a pair of surfaces, referred to as unstable and stable manifolds, that form the boundaries of field line trajectories when followed in the forward and reverse directions respectively. In an unperturbed, axisymmetric system the unstable and stable manifolds identically overlay each other forming the more failure smooth seperatrix structure. In a nonaxisymmetrically perturbed system, the 3D structure of this homoclinic tangle determines how field lines inside (confined) a manifold are topologically organized across the poloidally oscillating boundary region with respect to field lines outside (unconfined or open scrape-off layer) a manifold. An important distinction here is that some of the confined field lines residing inside the tangle boundaries hit material surfaces when one of the lobes of the tangle intersects a surface. The net result is that in addition to the usual scrape-off layer field lines, that are filled with plasma due to cross-field transport, there is another class of field lines that hit material surfaces. These field lines are connected directly into the hot plasma region and can have relatively short connection lengths. Thus, these field lines transport heat and particles from regions with very high plasma 
temperatures, in the interior of the plasma, directly to material surfaces. An example of one such field line is shown in Fig. 12(b), a Poincaré plot of the same data shown in Fig. 12(a) but mapped into $\psi_{N}$ versus poloidal angle $(\theta)$ space. The complex oscillations of the unstable invariant manifold around $\psi_{N}=1.0$ are quite clear in this representation. One field line of particular interest is represented by the "+" symbols. This field line intersects the divertor target when the tip of the homoclinic lobe (formed by the unstable manifold) cuts through the divertor plane near the location of the unperturbed inner strike point at $\theta \approx 255$ (deg.). We note that this field line samples plasma well inside the pedestal $\left(\psi_{N} \approx 0.88\right)$ and connects this plasma to the divertor target plate after only 93 toroidal revolutions (i.e., of order $0.85 \mathrm{~km}$ ).

We use data from calculations such as those shown in Fig. 12 to estimate the average length of field lines started on each flux surface between $\psi_{N}=0.8$ and $\psi_{N}=0.99$ with steps of $\Delta \psi_{N}=0.01$. Here, a field line is integrated through 200 toroidal transits or until it hits a material surface and its length, $L_{\|}(m)$, along with its magnetic diffusion coefficient, $D_{m}=\Delta r^{2} / 2 L_{\|}(m)$ where $\Delta r(m)$ is the radial distance traveled, are calculated and stored for further analysis. Field lines are started at 180 uniformly distributed points on each flux surface and results from each of these points are averaged on each surface to produce estimates of $\left\langle L_{\|}\right\rangle$and $\left\langle D_{m}\right\rangle$ as a function of $\psi_{N}$. Figure 13(a) shows the variation in $\left\langle L_{\|}\right\rangle$ with $\psi_{N}$ for discharge 123301, the case shown in Fig. 12, with a $3.2 \mathrm{kA}$ current in both the upper and lower I-coil loops. Here, only field lines that escape and hit solid surface are used to calculate $\left(L_{\|}\right)$. The upper curve in Fig. 13(a) shows the maximum field line length for each $\psi_{N}$ and the lower curve shows the minimum length. Figure 13(b) shows the fraction of field lines that hit a material surface from each $\psi_{N}$. A second curve, indicated with black solid circles and a thin solid line in the lower right-hand corner of Fig. 13(a), gives the value of $\lambda_{e}$ calculated from the data shown in Fig. 8 for the full 
I-coil case. We note that some of the field lines hitting material surfaces (typically of order $10-15 \%$ ) have lengths $L_{\|} \leq \lambda_{e}$.

We now look at the case with a $3.2 \mathrm{kA}$ current in only the lower I-coil loops (123300). A contour plot of the poliodal mode spectrum, similar that of Fig, 2(b), is shown in Fig. 14. We see that the higher $m$ resonant modes in the pedestal $10 \leq m \leq 13$ are about a factor of 2-3 smaller in amplitude than in the full I-coil case when compared to the data in Fig. 2(b) and that the resonance curve falls off more rapidly with decreasing $\psi_{N}$ inside $\psi_{N}=0.9$ in the $1 / 2$ I-coil case. Since the island widths scale as $\sqrt{\delta b_{r}^{(m / n)} B_{\phi}^{-1}}$, the change in the island widths when using only the lower I-coil loops on the resonant structure across the pedestal is rather modest as can be seen in Fig. 15 where the upper bars represent the island widths in the 1/2 I-coil case and the lower bars are reproduced from Fig. 3, the full I-coil case, in order to compare the differences between the two cases. Comparing the $\left\langle L_{\|}\right\rangle$for these two cases we find that the curves are similar but the $1 / 2$ I-coil case falls off less rapidly with $\psi_{N}$ and there are significantly less open field lines with lengths $L_{\|} \leq \lambda_{e}$ than in the full I-coil case. For example, the percentage of field lines hitting a material surface from $\psi_{N}=0.97 \rightarrow 0.99$ in the $1 / 2$ I-coil case is $57 \%$, $40 \%$ and $41 \%$ respectively compared to $73 \%, 58 \%$ and $52 \%$ shown in Fig. 13 for these flux surfaces. The length of the shortest field lines lost from these flux surfaces in the $1 / 2$ I-coil case is typically longer than in the full I-coil case. For example, going from $\psi_{N}=0.99$ to $\psi_{N}=0.97$ the shortest field lines are: $46.9 \mathrm{~m}, 61.2 \mathrm{~m}$ and $164.4 \mathrm{~m}$ respectively in the $1 / 2 \mathrm{I}$-coil case compared to $45.6 \mathrm{~m}, 48.6 \mathrm{~m}$ and $92.8 \mathrm{~m}$ in the full I-coil case.

A comparison of the average magnetic diffusion coefficient $\left\langle D_{m}\right\rangle$ for the full I-coil, the 1/2 I-coil and the no I-coil cases discussed above is shown in Fig. 16. The lower curve shows the variation in $\left\langle D_{m}\right\rangle$ for the no I-coil case where the C-coil and field-errors produce a rather modest level of stochasticity. Comparing the no I-coil to the $1 / 2$ I-coil 
(middle curve) case we see that there is smaller difference in these two from $\psi_{N}=0.96 \rightarrow 0.99$ than with the full I-coil (upper curve) case which shows a significant increase over this region. The largest difference between the no I-coil case and the two I-coil cases is near $\psi_{N}=0.93$ where there is more than an order of magnitude increase in $\left\langle D_{m}\right\rangle$. 


\section{DISCUSSION}

A typical plasma response found in the low $v_{e, \text { neo }}^{*}(0.12 \rightarrow 0.04) n=3$ edge resonant magnetic perturbation (RMP) experiments discussed above consists of a relatively strong decrease in $n_{e, p e d}$ profile with a modest increase or outward shift in the $T_{e, p e d}$ profile. Similar behaviors are seen in the ion profiles during these experiments [15]. This type of response is not easily explained using a straightforward application of collisionless stochastic magnetic field transport theory. Theoretically, the parallel thermal diffusivity $\chi_{e}$ is predicted to scale as $\mathrm{V}_{T e} D_{m}^{q l}$ in an isolated, strongly stochastic, layer containing a collisionless plasma [21]. Here, $\mathrm{v}_{T e}=\left(T_{e} / m_{e}\right)^{1 / 2}$ is the electron thermal velocity and $D_{m}^{q l}$ is a quasi-linear magnetic diffusion coefficient that is proportional to the square of the normalized magnetic perturbation $\delta b_{r}^{(m / n)} B_{\phi}^{-1}$ from each non-axisymmetric sources summed over adjacent resonant surfaces within the stochastic layer. $B_{\phi}$ is the toroidal magnetic field on axis. Since mass flow to a solid surface reaches the ion sound speed $\mathrm{c}_{s} \approx\left(2 T_{e} / m_{i}\right)^{1 / 2}$ at the electrostatic plasma-sheath boundary, an estimate of the parallel particle diffusivity through a stochastic field follows from $D_{\| p, s t o}=c_{s} D_{m}^{q l}$. Thus, to first order quasi-linear transport theory predicts that the particle loss rate should be about a factor of $\approx\left(m_{e} / m_{i}\right)^{1 / 2}$ smaller than the energy loss rate.

Using the data from Fig. 2(b) we estimate the quasi-linear stochastic magnetic field diffusivity as $D_{m, n}^{q l}(m)=\pi R_{0} \sum_{m=11-14}^{n=3} q_{m, n}\left(\delta b_{r}^{(m / n)} B_{T}^{-1}\right)^{2}(m)=3.5 \times 10^{-6}(m)$ for DIII-D discharge 123301 with $3.2 \mathrm{kA}$ in both the upper and lower I-coil loops. The magnetic diffusion coefficient $\left(D_{m}\right)$ calculated with the TRIP3D field line integration code is in good agreement with $D_{m, n}^{q l}$ at $\psi_{N}=0.95$ where we see from Fig. 16 that $D_{m}=3.9 \times 10^{-6}(\mathrm{~m})$ for the full I-coil case (upper curve). Thus, for $T_{e}^{p e d}=1.0 \mathrm{keV}$ (Fig. 8(b) upper curve), $\mathrm{V}_{T e}=1.4 \times 10^{7}(\mathrm{~m} / \mathrm{s})$, the cylindrical quasi-linear thermal diffusivity is $\chi_{m, n}^{q l}\left(\mathrm{~m}^{2} / \mathrm{s}\right)=v_{T e} D_{m, n}^{q l}\left(\mathrm{~m}^{2} / \mathrm{s}\right)=49\left(\mathrm{~m}^{2} / \mathrm{s}\right)$. Using $D_{m, n}^{q l}$ and the ion sound speed, the stochastic 
particle diffusivity across the pedestal is $\approx 2\left(\mathrm{~m}^{2} / \mathrm{s}\right)$. While experimentally the electron thermal transport data does indicate a modest $\sim 10-15 \%$ increase, as implied by the increase in the NBI hating power needed to maintain the same $T_{e, p e d}$ profile, this is significantly smaller than expected if $\chi_{e}$ were increased to $\approx 50\left(\mathrm{~m}^{2} / \mathrm{s}\right)$. In these plasmas the value of $\chi_{e, \perp}$ needed to match experimental $T_{e, p e d}$ profiles with those produced by 2D axisymmetric transport codes is $\approx 0.1 \rightarrow 0.4\left(\mathrm{~m}^{2} / \mathrm{s}\right)$ [29] so a $10-15 \%$ increase is well below the estimated increase due to quasi-linear transport.

In the models discussed here, the stochastic layer consists of open field lines that connect to solid surfaces which are mixed with closed field lines that appear to wander chaotically through the stochastic layer but do not hit a solid surface. Transport models that self-consistently include all the physics involved in this type of mixed edge stochasticity are inherently more complex than the quasi-linear formulation discussed above. As seen from Fig. 13, the lengths $L_{\|, c o n}$ of the open field lines connected to the solid surfaces ranges from several $\mathrm{km}$ to less than $50 \mathrm{~m}$. As the strength of the stochasticity increases, more field lines are connected to the surface from deeper inside the plasma and the fraction of the field lines with lengths shorter than the collisional mean free path $L_{\|, \text {con }} \leq \lambda_{e, s t o}$ increases. This is expected to increase the thermal transport and should reduce $T_{e, s t o}$ across the foot of the pedestal where $L_{\|, c o n} \leq \lambda_{e, s t o}$. Assuming a constant $n_{e}$ in this region, a reduction in $T_{e, s t o}$ reduces $\lambda_{e, s t o} \propto T_{e, s t}^{2} / n_{e}$ resulting in a flattened electron temperature profile near the foot of the pedestal which then leads to a stabilization of the drop in $T_{e, s t o}$. This flattening at the foot of the pedestal can produce an increase in the electron temperature gradient if $T_{e, p e d}$ at the top of the pedestal remains constant due to a constant flux of energy from the core plasma. This effect results in $T_{e, p e d}$ profiles that are similar to those measured across the transport barrier region of $\mathrm{H}$-mode plasmas. Effects such as these have been observed during stochastic boundary experiments in high $v_{e, \text {,neo }}^{*}$ limiter plasmas [30] and, as seen from the profile data shown 
above, are a common feature of the low $v_{e, \text { neo }}^{*} \mathrm{H}$-mode stochastic boundary experiments in DIII-D.

Thermal transport through these mixed stochastic layers is significantly more complex than envisioned by quasi-linear theory since the parallel electron heat conduction along the open field lines is very rapid. At the plasma-sheath boundary the parallel heat flux $q_{\|}=\gamma T^{1} T_{e}$ where $\gamma$ is a sheath transmission factor and $T^{1}$ is the particle flux impinging on the sheath $\left(T^{1}=n_{e} c_{s}\right)$. Thus, the overall transport scales as $T_{e}^{1 / 2}$ in the closed field line region and as $T_{e}$ in the open field line region in weakly collisional plasmas. In addition, the rate at which particles and energy are exchanged between these two regions is governed by the collisionality, the drifts and the fluctuation driven cross-field transport.

Finally, changes in the particle transport also affect the energy transport since the open field line conduction scales the fraction of open field lines hitting solid surfaces i.e., those with $L_{\|, c o n} \leq \lambda_{e, s t o}$ and $\lambda_{e, s t o}$ scales $T_{e, s t o}^{2} / n_{e}$. As noted above, the particle transport is more strongly affected than the energy transport with the I-coil. This can be seen by comparing changes in the $n_{e}$ and $T_{e}$ profiles such as those shown in Fig. 8(a) and $8(\mathrm{~b})$ respectively. The $n_{e}$ gradient region flattens and $n_{e, p e d}$ decreases in the full I-coil case compared to the $1 / 2$ and no I-coil case. The foot of the $n_{e}$ profile also increases with the I-coil. These effects are representative of an increase in the particle transport and are very suggestive of increased particle convection. At the same time we see that the $T_{e}$ gradient increases in the full I-coil case, Fig. 8(b), compared to the $1 / 2$ and 
no I-coil cases. These features are also reproduced during I-coil current scans [15] showing the same trend toward an increasing particle loss rate and increasing $T_{e}$ gradient with increasing I-coil current. Looking closely at the field line trajectory represented in Fig. 12 by the "+" symbols, we see an interesting feature. This field line was initially "stuck" on the surface of a rather large magnetic island at $q=3$. After $n_{56}$ toroidal transits from its starting position at $\psi_{N}=0.90,0=76 \mathrm{deg}$ it jumped (made a ballistic flight) to $\psi_{N}=0.98$ and within 37 toroidal transits struck the divertor target plate through the tangle lobe at $0=255 \mathrm{deg}$. Upon investigating this behavior it was found that a cluster of field lines with about the same starting position also has a similar behavior. This suggests the possibility of a rapid loss of energy along these field lines from the edge of the island and the formation of an electrostatic potential structure that could in principle enhance the radial $\vec{E} \times \vec{B}$ convective particle loss across the pedestal. Such a mechanism may be responsible for the large increase in the particle transport observed in the low $v_{e}^{*}$ experiments.

We also note from Fig. 12 that the way in which the open field line are connected to solid surfaces is topologically more complex due to the splitting of the axisymmetric separatrix. Nevertheless, it is quite encouraging that the pedestal profiles can be controlled with relatively small non-axisymmetric perturbations and that ELMs can be completely eliminated without destroying the H-mode transport barrier. Additionally, we see that by increasing the NBI heating power or the deuterium gas feed rate in the divertor we can increase the pedestal pressure without destabilizing large Type-I ELMs. 


\section{SUMMARY AND CONCLUSIONS}

In this paper we have shown that we can control the pedestal density and temperature profiles in ITER relavent, low collisionality, H-mode plasmas by applying small nonaxisymmetric resonant magnetic perturbations. These perturbations significantly change the global particle balance which then reduces the pedestal density gradient and modestly increases the pedestal temperature gradient. The net result of these changes is a reduction in the total pressure gradient across the pedestal region. In most cases we find that there is a strong correlation between the size of the resulting pressure gradient and the elimination of Type-I ELMs. The degree to which the pedestal profiles are changed is controlled by the current in the perturbation coil and the coil configuration used, the value of the safety factor at the $95 \%$ poloidal magnetic flux surface, the level of NBI heating power and the deuterium gas fueling rate in the divertor.

The experimental data show that the applied magnetic perturbations increases the particle transport significantly more than the energy transport. This contradicts expectations based on a straightforward application of quasi-linear stochastic transport theory and suggests that a more elaborate transport model is needed to interpret these experiments.

Results from field line integration modeling of the experiments show that the topology of the stochastic layer is significantly more complex than that envisioned by the standard transport theories. This increase in topological complexity comes about because of the fact the open field lines, having a rather broad range of lengths, are strongly mixed with closed field lines across most of the pedestal region. Calculations of the radial 
magnetic diffusion coefficient profile from the TRIP3D field line integration code have been compared with the quasi-linear magnetic diffusion coefficient and are found to be in very good agreement. These diffusion coefficients have been used to estimate a radial thermal diffusivity base plasma parameters matching the values use in these experiments. We find that the quasi-linear diffusion rate significantly overestimates that found in the experiments and have proposed a conceptual model that better matches the geometry used in the experiments.

In summary, these experiments have provided a wealth of new information about how complex stochastic boundaries affect the confinement and stability of low collisionality, poloidally diverted, H-mode plasmas and the promise that resonant magnetic perturbations hold for controlling pedestal region of the plasma. 


\section{ACKNOWLEDGMENTS}

This work was supported by the U.S. Department of Energy under DE-FC02-

04ER54698，DE-FG03-01ER54615，W-7405-ENG-48ח/ / 1 / [, DE-FG02-04ER54758,

' ( IFG02-89ER53297, DE-AC02-76CH03073, and DE-AC04-94AL85000. 


\section{REFERENCES}

[1] M. Okabayashi, J Bialek, M. S. Chance et al, Phys. Plasmas 8, 2071 (2001).

[2] E. Uchimoto, J. D. Callen, Z. Cheng and S. Prager Phys. Plasmas 1, 648 (1994).

[3] H. Takahashi, E. D. Fredrickson, M. J. Schaffer et al., Nucl. Fusion 44, 1075 (2004).

[4] M. Greenwald, R. Boivin, P. Bonoli et al., Plasma Phys. Control. Fusion 42, A263 (2000).

[5] K.H. Burrell, M.E. Austin, D.P. Brennan et al., Phys. Plasmas 8, 2153 (2001).

[6] A. Loarte, M. Becoulet, G. Saibene et al., Plasma Phys. Control. Fusion, 44, 1815 (2002).

[7] W. Horton, Rev. Mod. Phys. 71, 735 (1999).

[8] J.L. Luxon, et al., Nucl. Fusion 43, 1813 (2003).

[9] A. M. Garofalo, T. H. Jensen, L. C. Johnson et al, Phys. Plasmas 9, 1997 (2002).

[10] T.E. Evans, R.A. Moyer, and P. Monet, Phys. Plasmas 3 (2002) 4957.

[11] A. Loarte, G. Saibene, R. Sartori, et al., Plasma Phys. Control. Fusion, 45, 1549 (2003).

[12] T.E. Evans, R.A. Moyer, P.R. Thomas, et al., Phys. Rev. Lett. 92, 235003-1 (2004).

[13] T.E. Evans, R.A. Moyer, J.G. Watkins, et al., Nucl. Fusion 45, 595 (2005).

[14] R.A. Moyer, T.E. Evans, T.H. Osborne, et al., Phys. Plasmas 12, 056119 (2005). 
[15] K. H. Burrell, T. E. Evans, E. J. Doyle, et al., "ELM suppression in low edge collisionality H-mode discharges using $n=3$ magnetic perturbations" Plasma Phys. Control. Fusion (2005) submitted

[16] P.B. Snyder, H.R. Wilson, J.R. Ferron, et al., Phys. Plasmas 9, 2037 (2002).

[17] H.R. Wilson, P.B. Snyder, R.L. Miller and G.T.A. Huysmans, Phys. Plasmas 9, 1277 (2002).

[18] Osborne 05 EPS

[19] Moyer 05 H-mode

[20] T. E. Evans, K. H. Burrell, M. E. Fenstermacher, et al., "ELM control in low collisionality tokamak plasmas with $n=3$ magnetic perturbations" Phys. Rev. Lett. (2005) submitted.

[21] A.B. Rechester and M. N. Rosenbluth, Phys. Rev. Lett. 40, 38 (1978).

[22] G.L. Jackson, et al., in Proc. of 30th EPS Conf. on Controlled Fusion and Plasma Physics, St. Petersburg, Russia (2003) P-4.47 on CDROM.

[23] J.T. Scoville and R.J. La Haye, Nucl. Fusion 43, 250 (2003).

[24] R. Fitzpatrick, Phys. Plasmas 5, 3325 (1998).

[25] A. Reiman, M. Zarnstorff, D. Mikkelsen, et al., Nucl. Fusion 45, 360 (2005).

[26] A. Boozer, Phys. Plasmas 12, 092504 (2005).

[27] J.T. Scoville et al., Nucl. Fusion 31, 875 (1991). 
[28] T. E. Evans, R. K. W. Roeder, J. A. Carter, B. I. Rapoport, M. E. Fenstermacher and C. J. Lasnier, J. Phys.: Conf. Ser. 7 (2005) 174.

[29] G. Porter, et al.

[30] T.E. Evans, J.S. deGrassie, G.L. Jackson, et al., J. Nucl. Mater. 145-146, 812 (1987). 


\section{FIGURE CAPTIONS}

Fig. 1. (a) Plasma position used in the experiments discussed in this paper relative to the DIII-D lower divertor cryopump, the upper and lower I-coil segments (behind the graphite tiles inside the vacuum vessel - not shown) and the DIII-D $n=1$ field-error correction coil (C-coil) centered on the mid-plane outside the vacuum vessel. (b) 3D representation of the DIII-D I-coil configured for $n=3$, even parity (up-down symmetric), operations as used in the experiments discussed in this paper.

Fig. 2. (a) Contour plot of the combined Fourier mode amplitudes for each of the $n=1$ toroidal modes and (b) $n=3$ modes interacting with the outer flux surfaces in DIII-D discharge 123301 shown as a function of poloidal mode number $(\mathrm{m})$ and normalized poloidal flux $\left(\psi_{N}\right)$. The dashed $m=n q$ resonance line shows the location of the each discrete resonances (solid black dots) in $\psi_{N}$ for both the $n=1$ and $n=3$ perturbations.

Fig. 3. Vacuum magnetic island $(m / n)$ positions and widths plotted in terms of their safety factor $(q)$ versus normalized polodal flux $\left(\psi_{N}\right)$. With the exception of the $n=2$ islands, the widths are calculated using the mode amplitudes shown in Fig. 2.

Fig. 4. Time evolution of a typical $n=3$, even parity, discharge (122338) used in the low $v_{e, \text { neo }}^{*}$ RMP experiments showing: (a) the plasma current $I_{p}$, the safety factor at $\psi_{N}=0.95$ $q_{95}$ shifted down by 3 and the I-coil current, (b) the lower divertor $D_{\alpha}$ recycling signal, (c) the line integrated density $\bar{n}_{e}$, the pedestal density $n_{e, p e d}$ and (d) the neutral beam input power, "beams", along with the total "radiated" power from the plasma.

Fig. 5. (a) normalized pedestal temperature $\hat{T}_{e, p e d}$, density $\hat{n}_{e, p e d}$, pressure $\hat{p}_{e, p e d}$ and $q_{95}$ (b) a lower $D_{\alpha}$ recycling signal during discharge 122338 with $7.9 \mathrm{MW}$ of NBI heating power. 
Fig. 6 Comparisons of the: (a) $n_{e, p e d}$, (b) $T_{e, p e d}$ and (c) $p_{e, p e d}$ profiles averaged over $2.71 s \leq t \leq 3.11 s$ during the RMP assisted ELM-free state for two cases in shown in Figs. 5 and 6.

Fig. 7 Time evolution of the (a) $n_{e, p e d}$ profiles in discharges with no I-coil current (123302), current in the lower I-coil loops only (123300) and current in both the upper and lower I-coil loops (123301) as indicated by (b). Lower divertor $D_{\alpha}$ recycling signals showing ELM characteristics with no I-coil (c), the lower I-coil loops only (d) and both the upper and lower I-coil loops (e).

Fig. 8 A comparison of the: (a) $n_{e, p e d}$, (b) $T_{e, p e d}$ and (c) $p_{e, p e d}$ profiles averaged over $2.61 s \leq t \leq 3.01 s$ for case with no I-coil, the lower I-coil only and the full I-coil.

Fig. 9 Typical time evolution of an $n=3$, even parity, discharge used divertor gas puff comparisons. In this particular discharge (122489) the deuterium puffing rate in the private flux region is 8.0 Torr-liter/s. Shown here are: (a) $q_{95}$ shifted down by 3 and the I-coil current, (b) a lower divertor $D_{\alpha}$ recycling signal, (c) $\bar{n}_{e}, n_{e, p e d}$ and (d) the NBI heating power, "beams", along with the total "radiated" power from the plasma.

Fig. 10 Time evolution of the: (a) $n_{e, p e d}$ profiles in discharges with lower divertor gas puffing rates of 8.0 Torr-liter/s (lower curve - 122489), 20.4 Torr-liter/s (middle curve 122490) and 39.2 Torr-liter/s (upper curve - 122492) along with the (b). I-coil timing and lower divertor $D_{\alpha}$ recycling signals showing ELM behavior with puffing rates of: 8.0 Torr-liter/s (c), 20.4 Torr-liter/s (d) and 39.2 Torr-liter/s (e).

Fig. 11 Changes in the: (a) $n_{e, p e d}$, (b) $T_{e, p e d}$, (c) $p_{e, p e d}$ and (d) $\nabla p_{e, p e d}$ profiles averaged over $2.91 s \leq t \leq 3.1 s$ for lower divertor gas puffing rates of: 0.0 Torr-liter/s and no I-coil (a) upper curve, 39.2 Torr-liter/s with a $3.2 \mathrm{kA}$ I-coil current (a) $2^{\text {nd }}$ curve down, 
20.4 Torr-liter/s with a $3.2 \mathrm{kA}$ I-coil current (a) $3^{\text {rd }}$ curve down and 8.0 Torr-liter/s with a 3.2 kA I-coil current (a) lower curve.

Fig. 12 Poincaré plot of magnetic field line trajectories for discharge 123301 with a $3.2 \mathrm{kA}$ current in both the upper and lower I-coil loops showing (a) the R,Z coordinate representation with the DIII-D wall and unperturbed inner flux surfaces (dashed lines) and (b) the same data represented in $\psi_{N}$ versus poloidal angle. The "+" symbols show the position of a single field line, followed in the positive toroidal angel direction, with each toroidal revolution.

Fig. 13(a) Average, maximum (upper) and minimum (lower) field line length in kilometers calculated with the TRIP3D code for the case with a $3.2 \mathrm{kA}$ current in both the upper and lower I-coil loops (123301). The thick solid curves are for field lines followed in the forward direction and the dashed curves for field lines followed in the backward direction. The thin solid curve in the lower right-hand corner shows the collisional mean free path length for electrons $\lambda_{e}$ as a function of $\psi_{N}$. (b) the fraction of field lines lost from each $\psi_{N}$ surface.

Fig. 14 Contour plot of the poloidal Fourier mode amplitudes for a case with an $n=3$ current in only the lower I-coil loops during DIII-D discharge 123300 . The mode amplitudes are shown as a function of poloidal mode number $(\mathrm{m})$ and normalized poloidal flux $\left(\psi_{N}\right)$. The dashed $m=n q$ resonance line shows the location of the each discrete resonances (solid black dots) in $\psi_{N}$.

Fig. 15 Comparison of $n=3$ vacuum magnetic island $(m / 3)$ positions and widths plotted in terms of their safety factor $(q)$ versus normalized polodal flux $\left(\psi_{N}\right)$ for similar discharges with a $3.2 \mathrm{kA}$ current in both the upper and lower I-coil loops (lower bars 123301) and a $3.2 \mathrm{kA}$ current in only lower I-coil loops (upper bars -123300). 
Fig. 16 Magnetic diffusion coefficient $D_{m}(m)$ calculated with TRIP3D for a discharge with the $\mathrm{C}$-coil and field-errors only, i.e., no I-coil (lower curve), a repeat discharge where the lower loops of the I-coil were run with a current of $3.2 \mathrm{kA}$ (middle curve) and a repeat discharge with both the upper and lower I-coil loops using a current of $3.2 \mathrm{kA}$ (upper curve). 
(a)

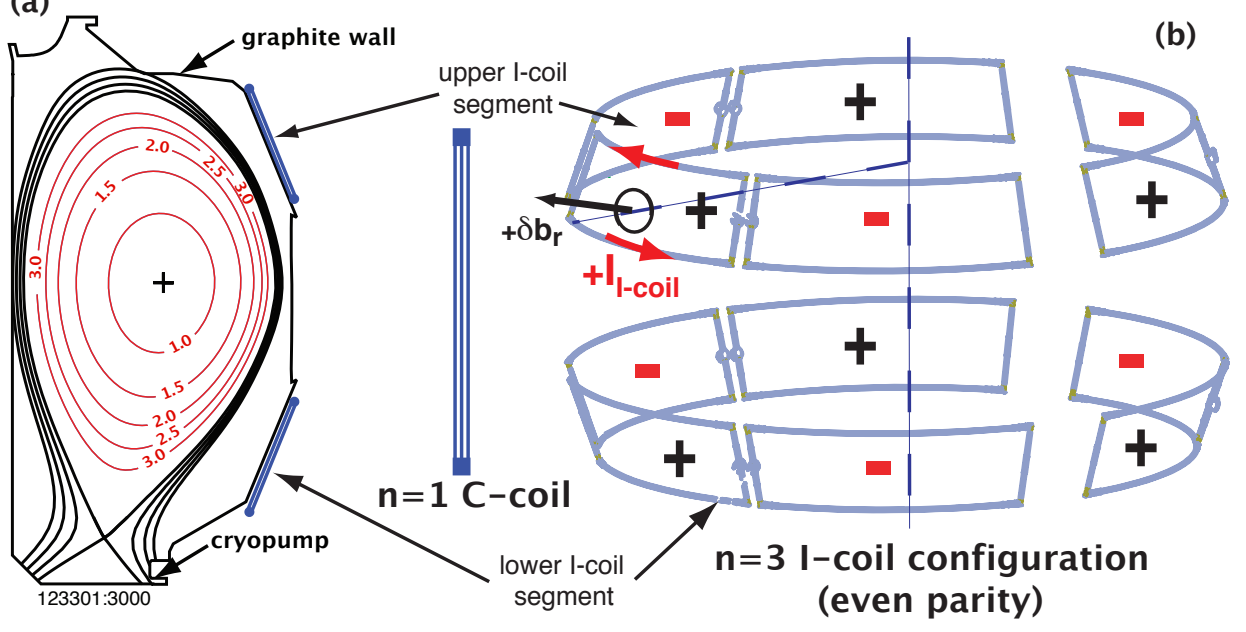

05 Evans Phys. Plasmas fig. 1 


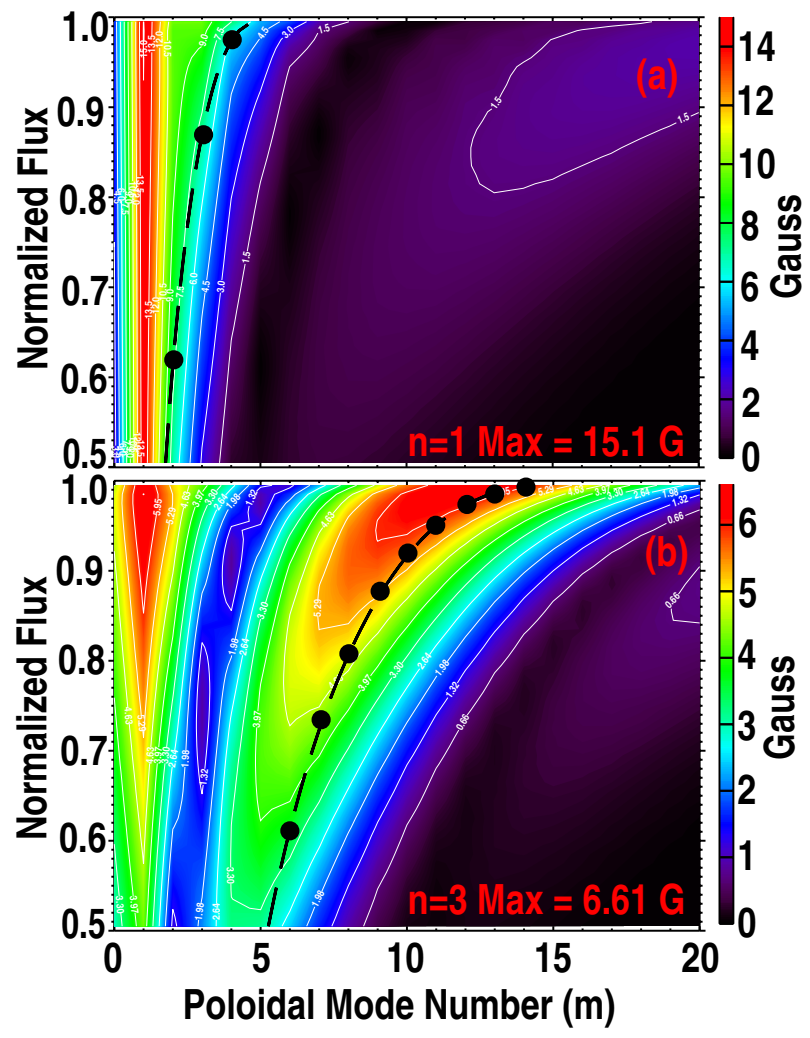

05 Evans Phys. Plasmas fig. 2 


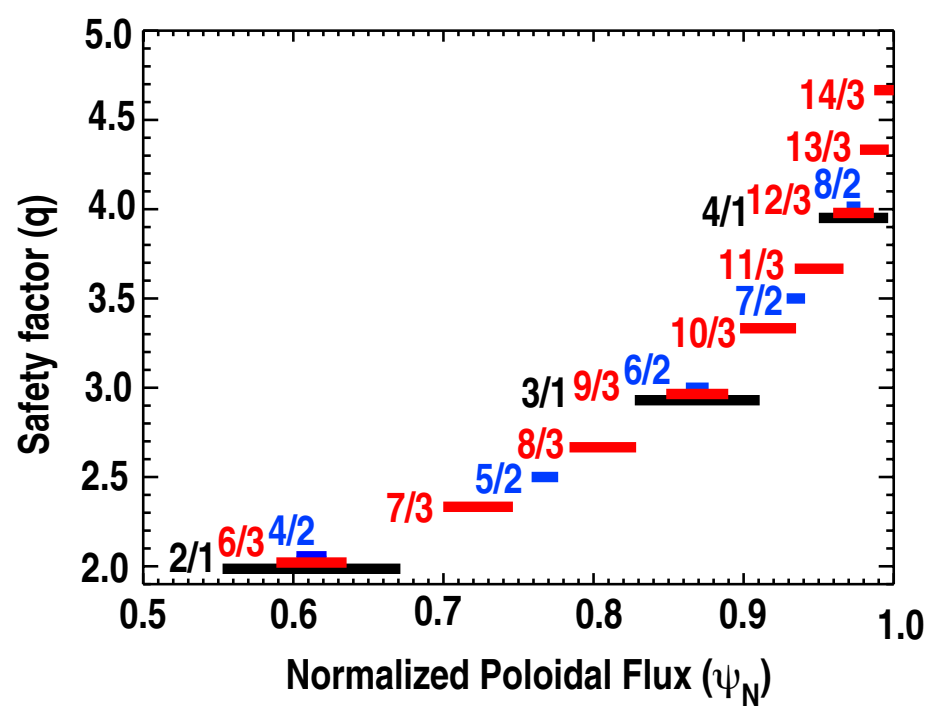

05 Evans Phys. Plasmas fig. 3 


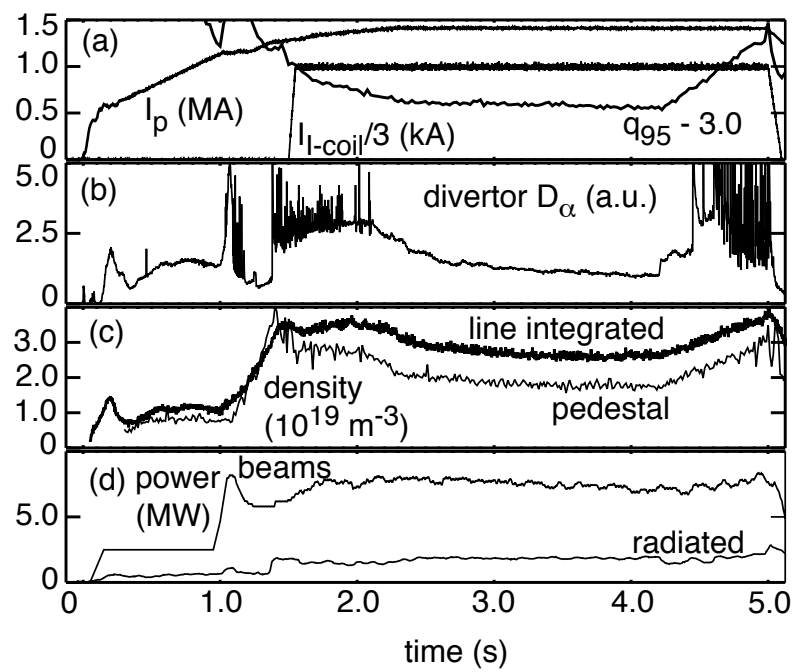

05 Evans Phys. Plasmas fig. 4 


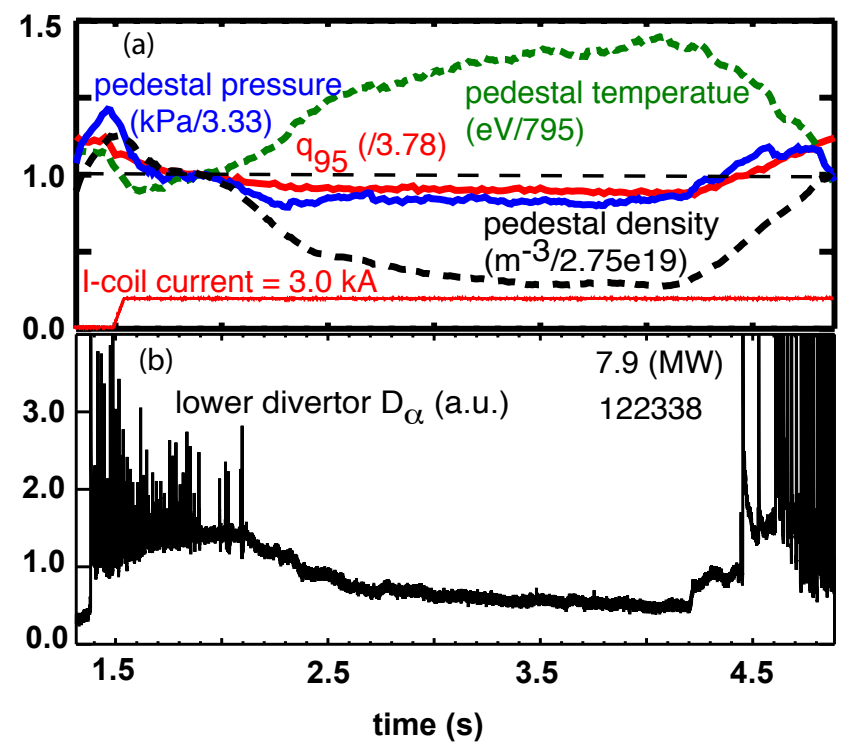

05 Evans Phys. plasmas Fig. 5 


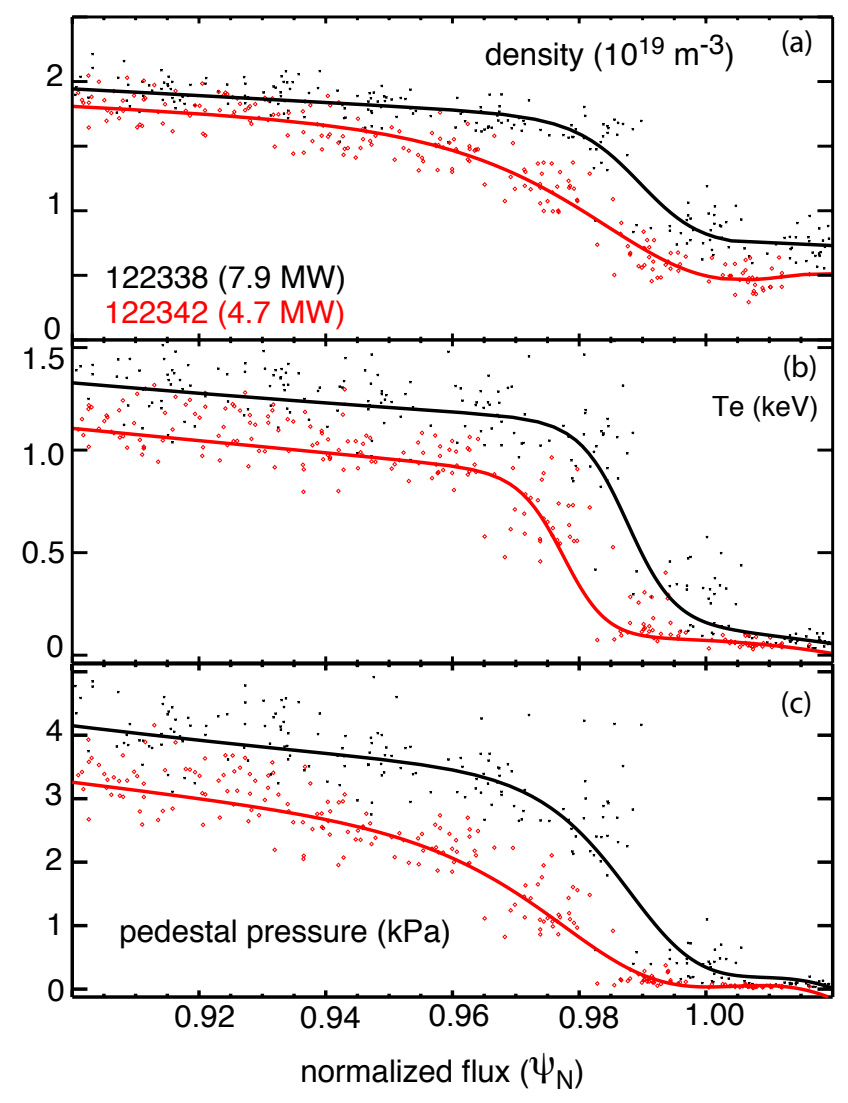

05 Evans Phys. Plasmas fig. 6 


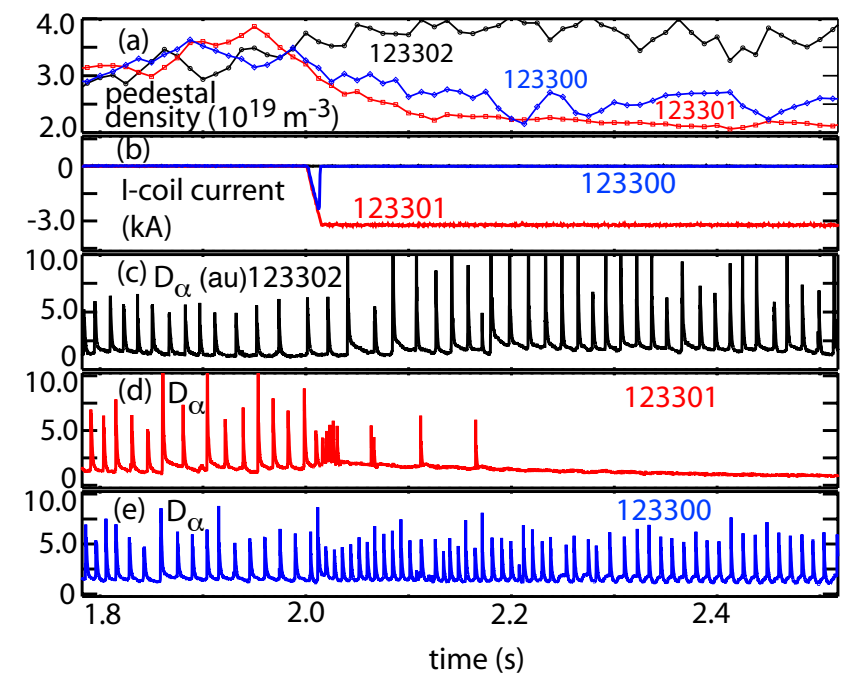

05 Evans Phys. Plasmas fig. 7 


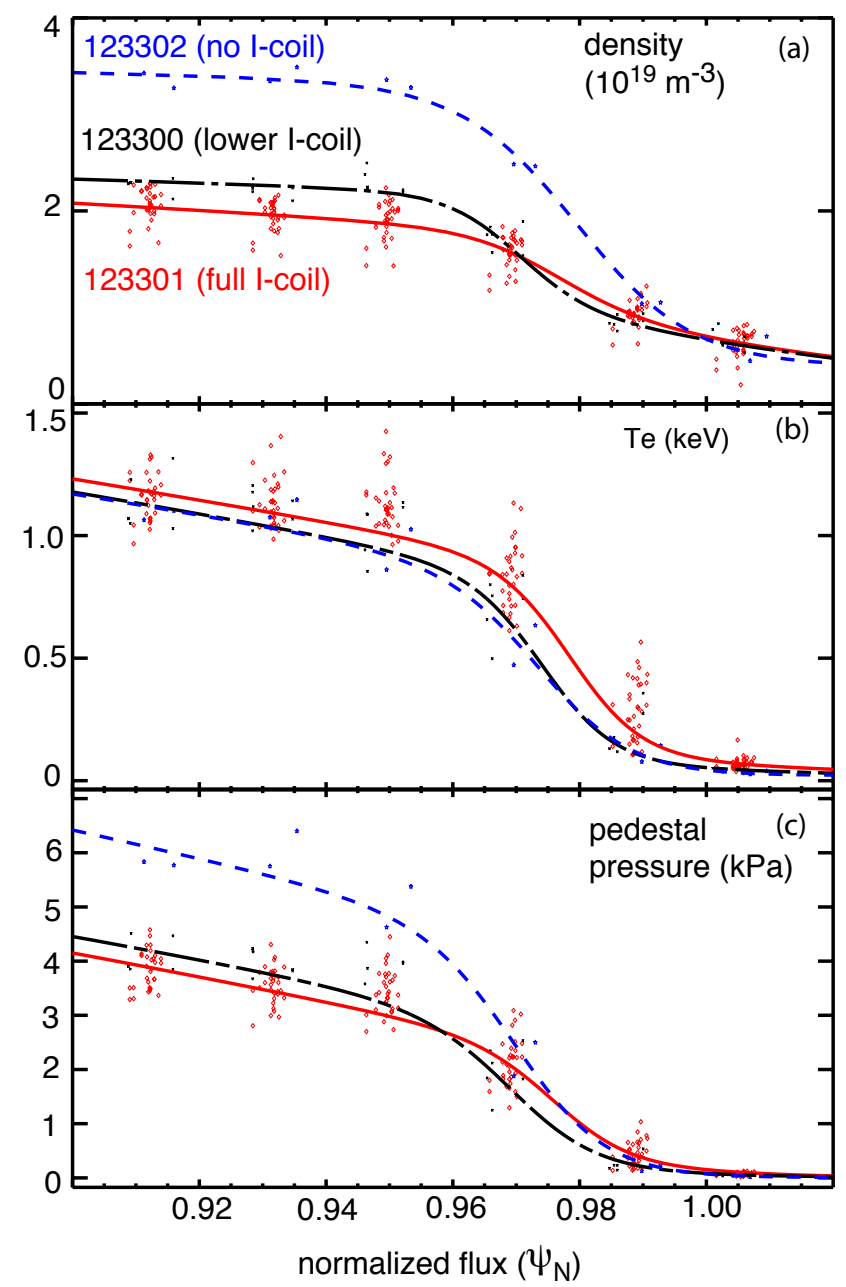

05 Evans Phys. Plasmas fig. 8 


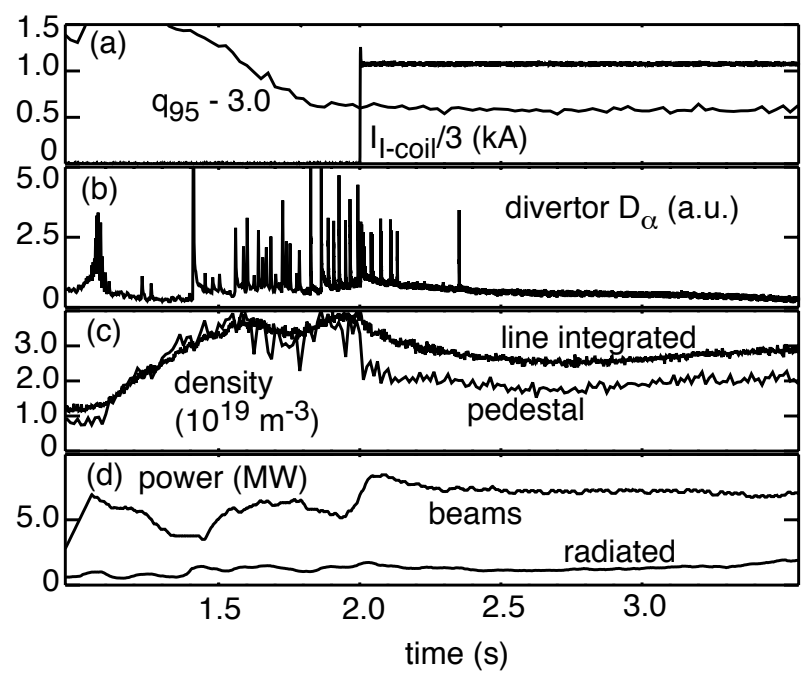

05 Evans Phys. Plasmas fig. 9

122489 


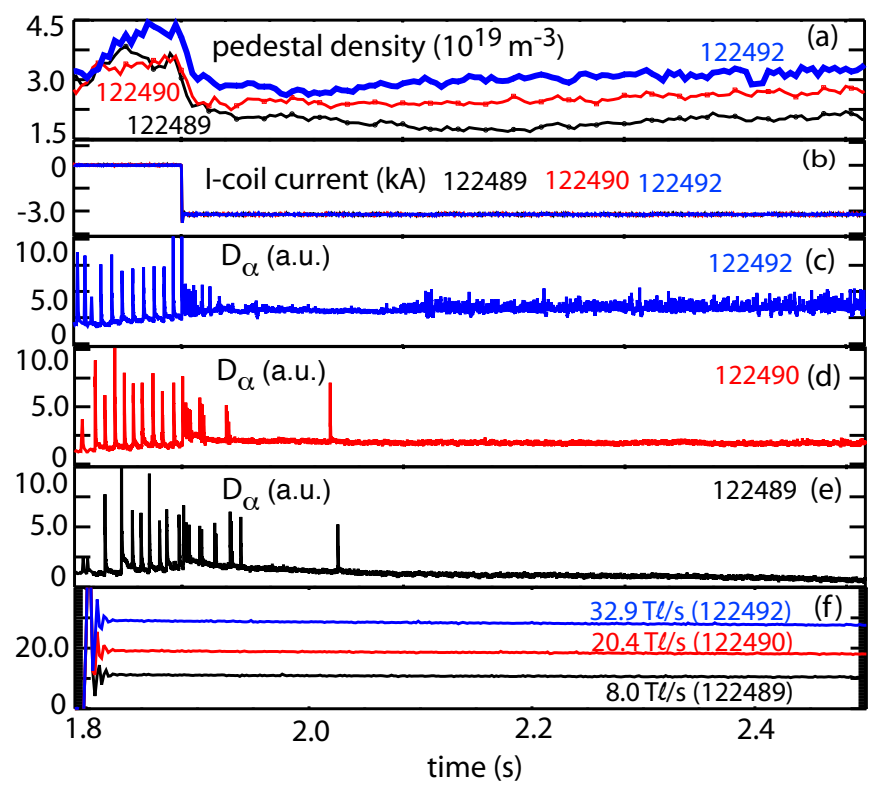

05 Evans Phys. Plasmas fig. 10 


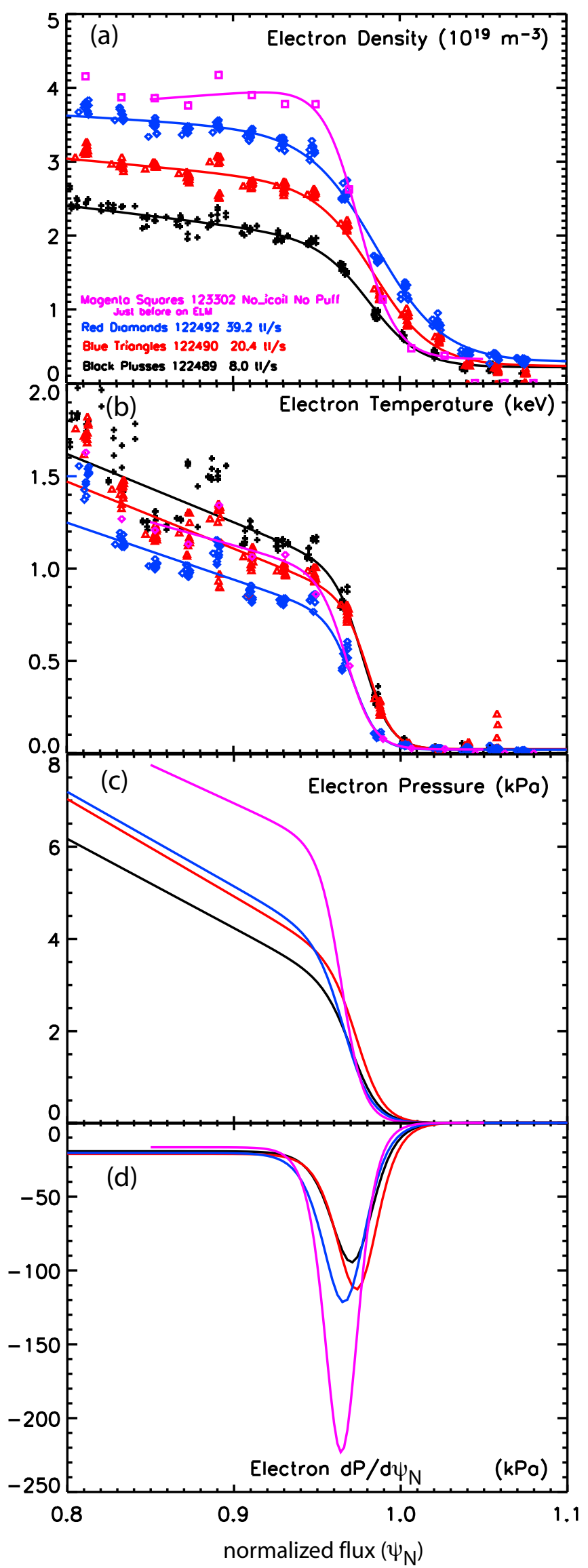

05 Evans Phys. Plasmas fig. 11 

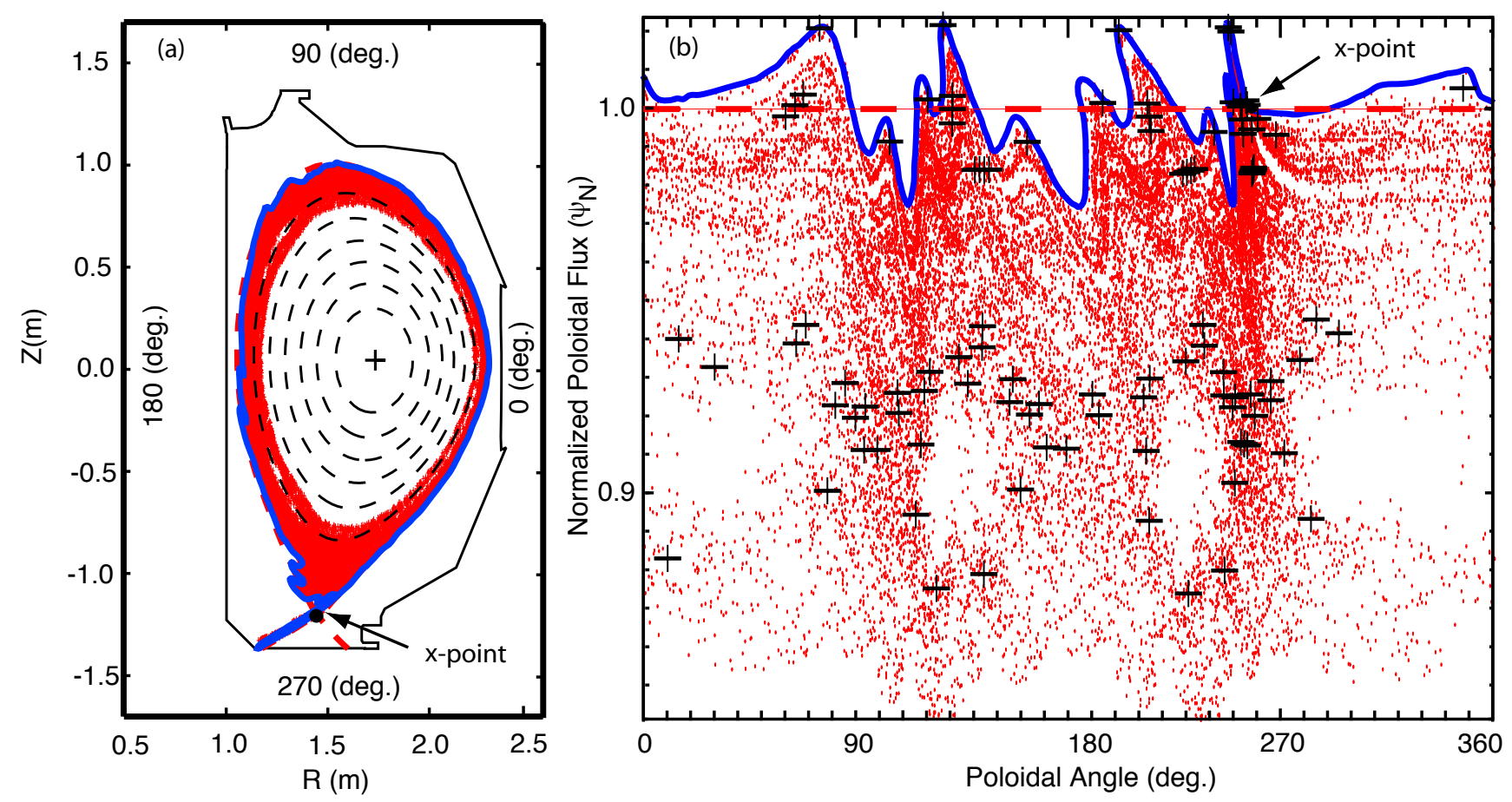

05 Evans Phys. Plasmas fig. 12

(double column wide) 


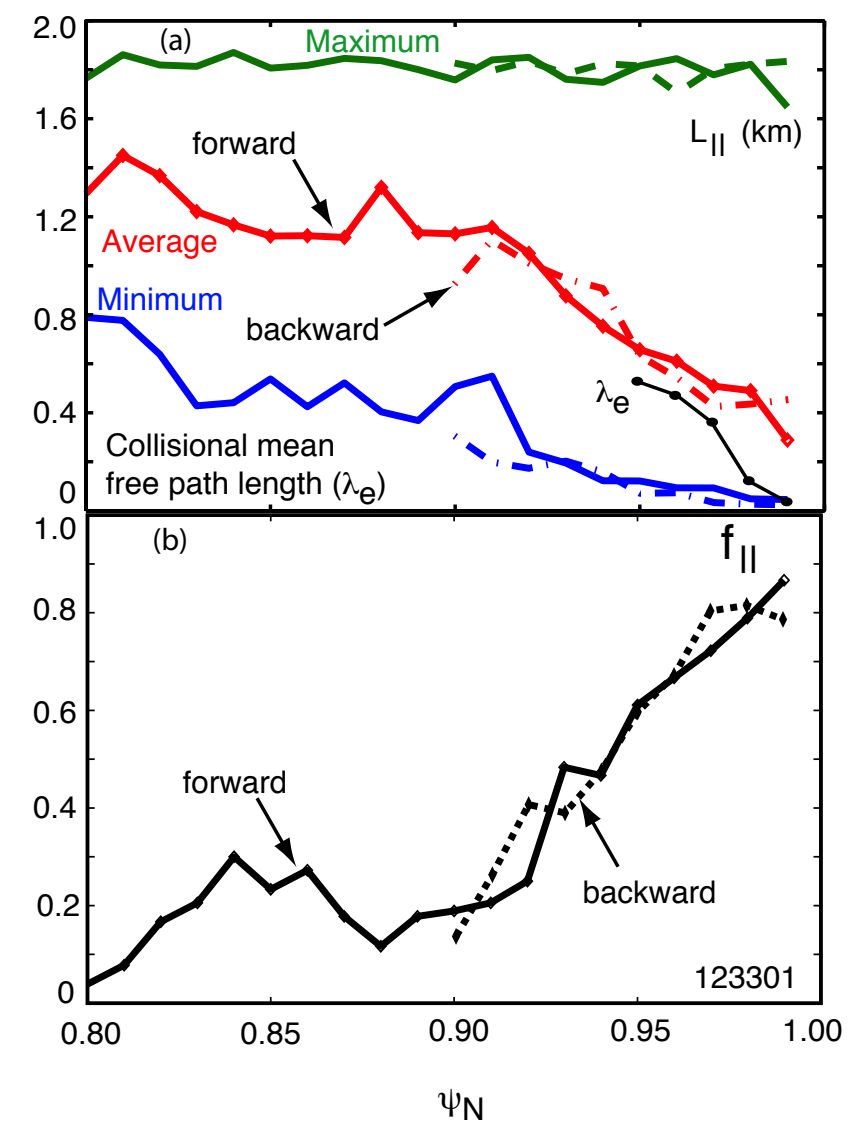

05 Evans Phys. Plasmas fig. 13

TRIP3D: Escape Length 1233013000 ms TRIP3D: Escape Fraction 1233013000 ms 


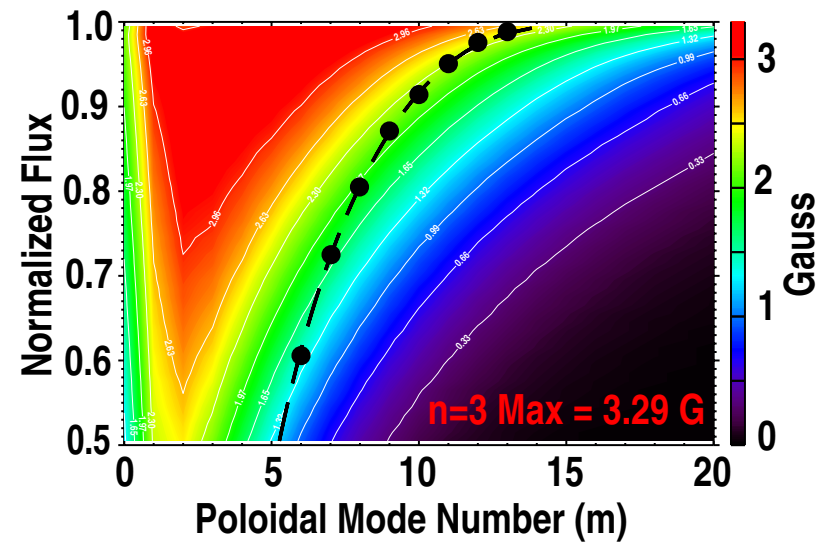

05 Evans Phys. Plasmas fig. 14 


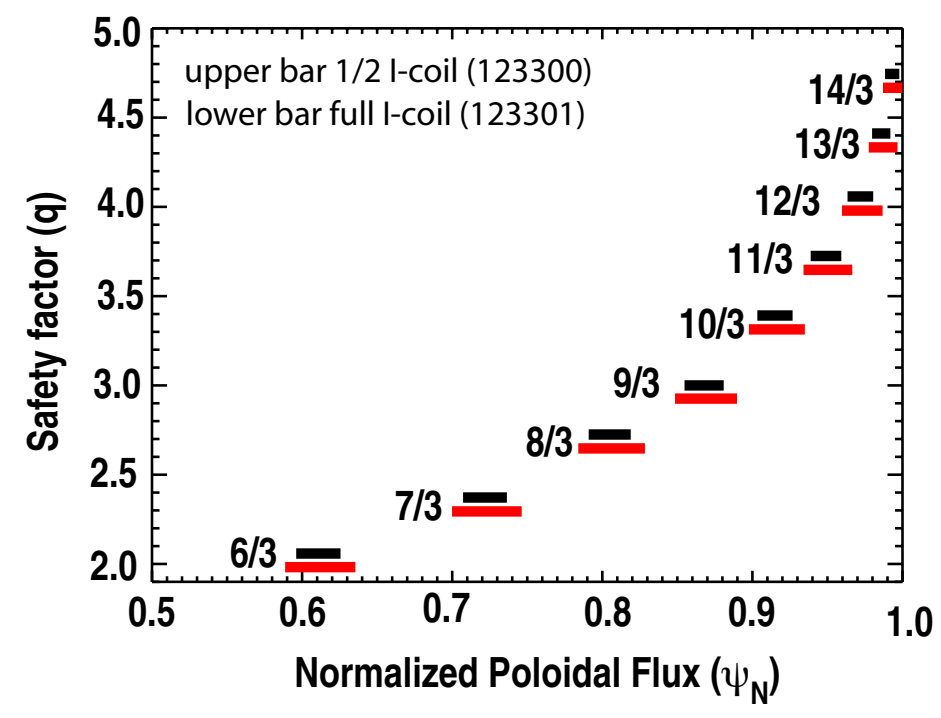

05 Evans Phys. Plasmas fig. 15 


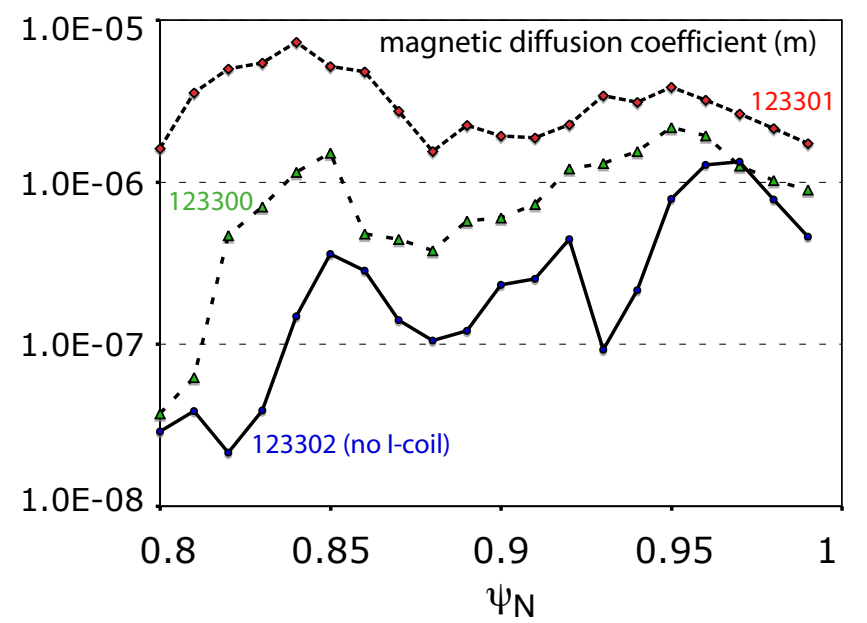

05 Evans Phys. Plasmas fig. 16 